\title{
Erratum to: Side-channel attacks on the McEliece and Niederreiter public-key cryptosystems
}

\author{
Roberto Avanzi · Simon Hoerder • Dan Page • \\ Michael Tunstall
}

Published online: 11 January 2012

(C) Springer-Verlag 2011

Erratum to: J Cryptogr Eng (2011) 1:271-281

DOI 10.1007/s13389-011-0024-9

S. Hoerder's name was erroneously added to the affiliation Qualcomm CDMA Technologies GmbH, Munich, Germany. The correct authors' affiliations are given below.

The online version of the original article can be found under doi:10.1007/s13389-011-0024-9.

R. Avanzi ( $\triangleleft)$

Qualcomm CDMA Technologies GmbH, Munich, Germany

e-mail: roberto.avanzi@gmail.com

S. Hoerder $\cdot$ D. Page $\cdot$ M. Tunstall

Department of Computer Science, University of Bristol,

Bristol, UK

e-mail: hoerder@compsci.bristol.ac.uk

D. Page

e-mail: page@compsci.bristol.ac.uk

M. Tunstall

e-mail: tunstall@compsci.bristol.ac.uk 